diese Pflanze, als dass er sich in derselben anhäufen könnte. Ein Vergleich mit dem frühern Versuche ergibt also den Schluss, dass gewisse indifferente Farbstoffe von e in ig e n Pflanzen allerdings auf organische Weise aufgenommen werden, und dass dieser Prozess nur vom chemischen Verhältnisse derselben zu den Farbstoffen abhängig gedacht werden kann.

P.

\title{
Hotanischer Tauselnverein in Wien.
}

Sendungen sind eingetroffen : 8. Von Herrn Gubernialrath Dr. Stre inz in Grala mit Pflanzen aus verschiedeneu Floren. - 9. Von Herrn Plus k a in Lomnitz mit Pflanzen aus der Flora daselbst, darunter auch Cryptogamen. - 10. Von Herrn Major G e g e nba u er in Gralz mil Pflanzen aus Sleiermark und ltalien. - Sendungen sind abgegangen an die Herren: $B$ a le $k$ in Fünflirchen und $\mathrm{M}$ a $\mathrm{y} r$ in Wien.

Das Verzeichniss der nea eingelaufenen Arten wird erscheinen, sobald die Rüclistände zum grössien Theile berichtiget sein werden.

\section{Gärten in Wien.}

Im botanischen Garten blühen derzeit:

Chamaedorea elatior, - Genista candicans L in n. - Genista laburnoides - Mercurialis zeyheriana K z e. - Pittosporum lanceolatum $\mathrm{P} u ̈ \mathrm{t}$ e $\mathrm{Pl}$ i $\mathrm{ch}$. - Pittosporum ferrugineum. A it. - Phillyrea latifolia L i n $\mathrm{n}$. - Rhamnus Ataternus $\mathrm{L}$ i $\mathrm{n}$. - - Ribes sanguineum $\mathrm{P}$ u's $\mathrm{ch}$. - R. glutinosum $\mathrm{B}$ o $\mathrm{t} \mathrm{b}+-$

$[\tilde{5}]$

\section{I $S \&$ \& a t}

\section{Jahrbuch der Handwirthschaft.}

Herausgegebeu v. W. Löbe. III. Jahrg. 24 Bogen. Preis geheftet Thir. 2. -

Die besondere Brauchbarkeit dieses Jahrbuches ist Seitens des hohen k. k. Ministeriums für Landescultur in Wien bereits lobend anerkannt, und es ist in Folge dessen dasselbe Seitens dieser hohen Behorde allen Iandwirthschafulichen Vereinen, Lehranslalten u. s. w. der österreichischen Monarchie zur Anschaffung empfohlen worden, worauf wir die jenseitigen verehrlicheu Vereine noch besonders aufmerksam machen.

Unser Jahrbuch ist in der That eines der reichballigsten Nachschlagebiicher und vereinigt in vielen hundert systematısch geordneten Nachweisen alle praktisch erprobten oder sonstwie bemerkenswerihen Notizen über neue Betriebsweisen, Verbesserungen und Fortschritte, von welchen der strebsame Landwirth entweder erst sehr spät Kenntnsss erlıalt, oder die zum grössten Theile, bei ihrer Zerstreutheit in hunderten von in- und ausländischen Blättern, für das praklische Leben ganz verloren gehen.

Was das Jahrbuch gibi, das gibt es übersichtlich, nach Fächern geordnet, systemalisch. Ein alphabetisch geordnetes Inhalisverzeichniss erleichtert das Aufsuchen und Nachschlagen sehr.

Jeder Jahrgang enthält circa 800 Aufsätze, Notizen, statistische und andere Miltheilungen u. s. $w$.

Wir wenden uns mil uuserm Lnternehmen zunächst an die gesammten landwirthschaftlichen Vereine und deren Träger, weil wir voraussetzen, dass für diese vorzugsweise dieses Jahrbuch ganz unentbehrlich, ja dass es gewissermassen ihre Pflicht sei, das Forterscheinen jenes für die Landwirthschaft hochwichtigen Werkes durch dessen Unterstützung zu sichern. Circulirt das Jahrbuch unter den enzelnen Vereinsmilgliedern, so erhalten sie alle Kenntniss vom Inhait desselben, und nach vollendetem Umlaufe bleibt das praktische Buch immer noch ein schätzenswerthes Werk in der Vereinsbibliothek, ein Werk, das nie veraltet.

Die Redaction des Jahrbuches

W. L ö b e.

Die Verlagshandlung 0 . S p a m e r. Leipzig. Querstr. Nr. 27. d.

Redacteur und Herausgeber: Alex. Skofit $z_{0}--$ Druek von c. Ueberreuter. 